\title{
Medizin und Recht. Grenzziehungen und Grenzüberschreitungen
}

\begin{abstract}
I.
Das Verhältnis von Medizinern und Juristen ist ein ambivalentes. Medizinische und Juristische Fakultäten gehören zu den - um ein Wort des Wissenschaftsrates in seiner jüngsten Stellungnahme zur Rechtswissenschaft $^{1}$ aufzugreifen - sog. Professionsfakultäten. Die Angehörigen beider Professionen bedürfen gelegentlich der Hilfe der jeweils anderen, würden aber jeweils wohl am liebsten nie in eine solche Situation der „Bedürftigkeit“" geraten. Nicht zuletzt deshalb sucht der erkrankte Jurist nicht selten spät, vielleicht zu spät den Mediziner auf, und umgekehrt steht es nicht anders. Das alles unterscheidet Mediziner und Juristen freilich noch nicht von Nicht-Medizinern
\end{abstract}

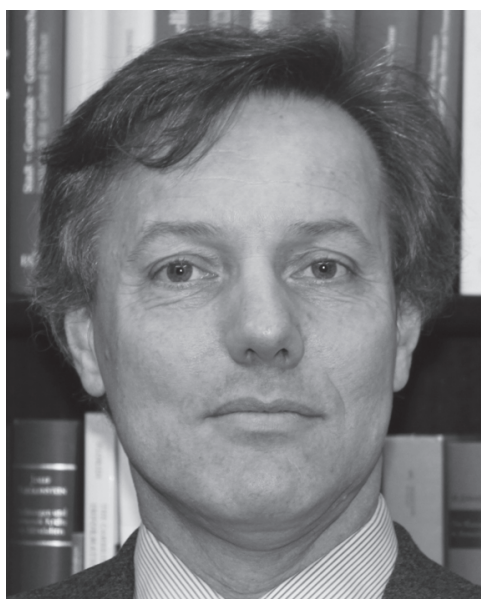

Andreas Spickhoff, Professor für Bürgerliches Recht, Medizinrecht, Internationales Privatrecht und Rechtsvergleichung an der Georg-AugustUniversität Göttingen, O. Mitglied der Göttinger Akademie seit 2012 oder Nicht-Juristen. Was macht also das spezifische Verhältnis, vielleicht Spannungsverhältnis, zwischen Ärzten und Juristen (jedenfalls manchen) aus?

Ein Grund dafür liegt vermutlich in dem Umstand, dass es das reflektiert als solches verstandene Rechtsgebiet des Medizinrechts noch nicht lange gibt. Selbst der Begriff "Medizinrecht“ (mit seinem wesentlichen Bestandteil des Arztrechts) ist bis heute noch nicht ganz deutlich festgelegt. Fast synonym zu ihm wird der Begriff Gesundheitsrecht verwendet. Misserfolge der Ärzte wurden im Wesentlichen vom Recht bis weit in das

\footnotetext{
1 Perspektiven der Rechtswissenschaft in Deutschland. Situation, Analysen, Empfehlungen (vom 9. November 2012), S. 5, 7.
} 
19. Jahrhundert herein - sieht man einmal von Vorsatzdelikten $a b-a l s$ schicksalhaft akzeptiert ${ }^{2}$. An den Juristischen Fakultäten der Universitäten hat sich - nicht nur in Deutschland - das Medizinrecht als eigenständige Disziplin erst in den letzten Jahrzehnten zu etablieren begonnen ${ }^{3}$. Dabei wird man sagen dürfen, dass das Arzt- bzw. Medizinrecht insbesondere hier in Göttingen durch Hans-Ludwig Schreiber und Erwin Deutsch seit den 70er Jahren des vergangenen Jahrhunderts als mittlerweile im Wesentlichen akzeptiertes besonderes Rechtsgebiet eingeführt worden ist; der „Dritte im Bunde" derer, die maßgeblich dazu beigetragen haben, war Adolf Laufs in Heidelberg. Auf diese Weise bin auch ich, insbesondere über eine Mitarbeit am damaligen Lehrstuhl von Erwin Deutsch, zum Medizinrecht gelangt. Der Umstand, dass Großvater, Onkel und Cousin Ärzte (alle Gynäkologen) geworden sind, mag mein Interesse an diesem neuen Rechtsgebiet begünstigt haben.

Noch zu Beginn der 90er Jahre (und an den meisten Juristischen Fakultäten vielleicht auch heute noch) war es indes durchaus unter dem Aspekt einer späteren Berufbarkeit nicht ohne weiteres empfehlenswert, sich allzu sehr mit dieser an sich sehr zukunftsträchtigen, aber doch speziellen Materie - manche sagen auch: einem Orchideenfach - zu befassen. Es lag daher nahe, dass ich nach einer Dissertation zu einer Grundfrage des Internationalen Privatrechts (dem Rechtsgebiet, das uns sagt, wessen Staates Rechtsordnung in Fällen mit Auslandsberührung anwendbar ist) ${ }^{4}$ ein der Arzthaftung zwar nahestehendes, aber doch allgemein gehaltenes haftungsrechtliches Thema als Gegenstand der Habilitationsschrift aufgriff5.

Die zunehmende Verrechtlichung des Medizinrechts, die leider regelmäßig politischem Aktionismus und keineswegs (rechts-) wissenschaftlichen Vorarbeiten folgt, also nicht wirklich „Schuld“ der Juristen ist, hat die Skepsis von Medizinern gegenüber Juristen gewiss beflügelt. Keineswegs zu Unrecht wird sie als Ausdruck von wachsendem Misstrauen gegenüber Medizinern angesehen. Es sei erlaubt, das Ausmaß der Verrechtlichung der

2 Überblick zur Entwicklung bei Theresa Riegger, Die historische Entwicklung der Arzthaftung, Diss. iur. Regensburg 2007, S. 33-97; Eva Schumann, De medicis et aegrotis - Arztrecht im Frühmittelalter, Medizin und Haftung, Festschrift für Erwin Deutsch, 2009, S. 545, 556 f; Andreas Deutsch, Zwischen deliktischer Arzthaftung und Wetterzauber - Medizinrechtliche Fragestellungen im Klagspiegel um 1436, in: Humaniora, Festschrift für Adolf Laufs, 2006, S. 45 , 50 ff.; Gerhard Köbler, Vom Arzt im Recht zum Arztrecht, in: Humaniora, Festschrift für Adolf Laufs, 2006, S. $157 \mathrm{ff}$.

3 Zu Göttinger Vorläufern Erwin Deutsch, Arztrechtler in Göttingen: Ludwig von Bar, Ernst Rabel und Eberhard Schmidt, in: Rechtswissenschaft in Göttingen, Göttinger Juristen aus 250 Jahren (Hrsg. Fritz Loos), 1987, S. 289 ff.

4 Andreas Spickhoff, Der ordre public im Internationalen Privatrecht, 1989.

5 Andreas Spickhoff, Gesetzesverstoß und Haftung, 1998. 
Medizin an zwei herausgegriffenen Beispielen deutlich zu machen: In der ersten Auflage (1977) hatte das „Arztrecht“ von Adolf Laufs ein Volumen von 110 Seiten. Die sechste Auflage (2009) ${ }^{6}$ ist - bei gleich kleinem Drucktyp, aber deutlich größerem Format - 531 Seiten lang geworden. Ähnlich steht es mit dem Lehrbuch zum Medizinrecht, das von Erwin Deutsch begründet und seit der 5 . Auflage von mir mitbetreut wird. Es hat in der sechsten Auflage (2008) die 1000-Seiten-Grenze durchbrochen, während sich die erste Auflage $1983^{7}$ mit 352 Seiten begnügen konnte.

Besonders deutlich wirkt die Überregulierung im Bereich der Medizin nicht nur im Kontext der Arzthaftung, sondern auch etwa im Bereich der medizinischen Forschung, im Medizinprodukte- und Pharmazierecht und insbesondere im Recht der gesetzlichen Krankenversicherung. Diese Entwicklungen beruhen indes nicht allein auf übermäßigem Misstrauen gegenüber der Medizin und den sie tragenden Berufsgruppen und Unternehmen. Hinzu gekommen ist vielmehr eine Akzentverschiebung, die wohl auch ärztlicherseits mittlerweile konsentiert wird: Die Wahrung des Selbstbestimmungsrechts der Patienten ist in den Vordergrund gerückt worden. Der früher wohl stärker als heute anzutreffende ärztliche Paternalismus ist zurückgedrängt worden. Das ist mehr als eine bloße Gegenreaktion darauf, medizinisch ungünstige Verläufe per se als schicksalhaft zu akzeptieren.

Während bis weit in das 19., ja 20. Jahrhundert der Satz „salus aegroti suprema lex" anerkannt war, streitet man sich heute darüber, ob man nicht besser von „voluntas aegroti suprema lex“ spricht. Das Erfordernis des „informed consent" ist dabei wohl erst in der zweiten Hälfte des 19. Jahrhunderts entwickelt worden. Ein Zeuge ihrer Entwicklung ist der Göttinger Alumnus Bismarck ${ }^{8}$. Er berichtet über den an Kehlkopfkrebs erkrankten Thronfolger, den nachmaligen Kaiser Friedrich III.: „Die behandelnden Ärzte waren Ende Mai 1887 entschlossen, den Kronprinzen bewusstlos zu machen und die Exstirpation des Kehlkopfs auszuführen, ohne ihm ihre Absicht angekündigt zu haben. Ich erhob Einspruch, verlangte, dass nicht ohne die Einwilligung des Patienten vorgegangen und, da es sich um den Thronfolger handele, auch die Zustimmung des Familienhauptes eingeholt werde. Der Kaiser, durch mich unterrichtet, verbot, die Operation ohne Einwilligung seines Sohnes vorzunehmen“. Kurz darauf, am 31. Mai 1894, entschied das Reichsgericht ${ }^{9}$ den Fall eines Oberarztes, der unter Anklage der Körperverletzung stand. Er hatte an einem 7-jährigen Kind eine

\footnotetext{
Nunmehr gemeinsam verfasst von Adolf Laufs; Christian Katzenmeier; Volker Lipp, Arztrecht. Damals noch (nunmehr als teilweiser Untertitel dienend) „Arztrecht und Arzneimittelrecht“. Gedanken und Erinnerungen, Band II, 1898, S. 306.

Entscheidungen des Reichsgerichts in Strafsachen, RGSt 25, 375.
} 
Fußamputation vorgenommen, da eine tuberkulöse Vereiterung des Fußwurzelknochens vorlag. Der Vater des Kindes war ein Anhänger der Naturheilkunde und grundsätzlicher Gegner der Chirurgie. Er hatte der Operation widersprochen. Das Gericht sah in dem medizinisch indizierten Eingriff eine Körperverletzung, die nur durch die Einwilligung des sorgeberechtigten Vaters oder eines Pflegers gerechtfertigt werden könne.

Aus diesen Anfangsgründen ist neben der Einwilligung auch die Aufklärungspflicht zur Grundlage einer gefürchteten Haftung geworden, ja, nach der Ansicht vieler denaturiert: Zahlreiche Verfahren, in denen die Verletzung der Aufklärungspflicht gerügt wird, stellen in Wirklichkeit Kunstfehlerprozesse dar, bei denen die Verfehlung des medizinischen Behandlungsstandards zwar nicht bewiesen werden kann, aber doch nahe liegt oder zumindest vom Patienten vermutet wird. Hat sich das Risiko der Operation (auch in Form eines nicht nachweisbaren Behandlungsfehlers) verwirklicht, begründet schon die unzureichende Aufklärung über das Risiko prinzipiell die Haftung.

\section{II.}

Die beiden soeben angedeuteten großen Kontrollfelder in Bezug auf ärztliches Verhalten - fachgerechte Behandlung und Achtung der Patientenautonomie - bergen die Besonderheit in sich, dass die Frage, ob ein Behandlungsfehler vorgelegen hat, regelhaft ebenso wenig von einem Juristen alleine entschieden werden kann wie z. B. die Frage, ob ein Risiko bestanden hat, über das aufgeklärt werden musste. Juristen mischen sich zwar in praktisch jeden Lebensbereich ein, genauer: Sie werden geradezu um Einmischung ersucht, zumindest von einem der Beteiligten. Das führt zu einer anderen Facette des Untertitels meines Vortrags, der keineswegs nur juristische Grenzen der Medizin (von denen bisher eher die Rede war), sondern vielmehr auch Grenzen der juristischen Zunft anreißen möchte.

Was ist ein Behandlungsfehler, welche Risiken dürfen bei der Behandlung gelaufen werden, und über welche solcher (keineswegs notwendig von vorneherein zu einem Behandlungsfehler führenden) Risiken oder mögliche Alternativen ist der Patient aufzuklären? Diese Frage können Juristen im Prozess regelmäßig nicht ohne Hilfe, prozessrechtlich ausgedrückt: nicht ohne den Sachverstand der Medizin(er), beantworten. Dabei ist im Prozess die Grenzziehung zwischen richterlicher Tätigkeit und der Aufgabe von Sachverständigen, die bekanntermaßen oft nicht ganz zu Unrecht als „heimliche Richter" bezeichnet werden, theoretisch im Ausgangspunkt 
scheinbar einfach, in der Konkretisierung indes voller Fallstricke. Der Ausgangspunkt wird für den Zivilprozess recht einfach so ausgedrückt: „Da mihi factum, dabo tibi ius“. Für Rechtsregeln, für Normen und für darauf bezogene Wertungen ist der Richter zuständig, für die tatsächlichen Grundlagen der Subsumtion und ihre Einführung in den Prozess die Parteien, Letztere im Arzthaftungsprozess freilich nur in einer abgemilderten Form; im (deutschen) Strafverfahren besteht grundsätzlich eine Beibringungspflicht oder -obliegenheit gar nicht ${ }^{10}$.

Bezogen auf unsere Frage nach der Grenzziehung zwischen den Aufgaben von Juristen (als Richter) und Medizinern (als Sachverständige) bilden folgende Normen den Rahmen für die Aufgabenverteilung: $\$ 276$ Abs. 2 BGB besagt, dass fahrlässig handelt, wer die im Verkehr erforderliche Sorgfalt außer Acht lässt. Es kommt also weder auf die übliche Sorgfalt (den eingerissenen Schlendrian) an, noch - jedenfalls im zivilen Haftpflichtprozess - darauf, ob dem potentiell Haftpflichtigen die Einhaltung der normativ erforderlichen Sorgfalt auch subjektiv-individuell möglich war (was man sich bei demjenigen fragen könnte, der seine Examina nur mit Glück bestanden hat und so dumm ist, nicht einmal wissen zu können, wie wenig er weiß, den also nicht einmal ein - individuelles - Übernahmeverschulden trifft). Man spricht insoweit vom objektiv-typisierten Sorgfaltsmaßstab. Konkretisiert für den Bereich der Arzthaftung formuliert es das soeben verabschiedete Patientenrechtegesetz, das einen neuen Typus des „Behandlungsvertrages" in unsere zivilrechtliche Kodifikation, das Bürgerliche Gesetzbuch, introduziert, folgendermaßen ( $\$ 630$ a Abs. 2 BGB):

„Die Behandlung hat nach den zum Zeitpunkt der Behandlung bestehenden, allgemein anerkannten fachlichen Standards zu erfolgen, soweit nicht etwas anderes vereinbart ist."

Die fachlichen Standards werden namentlich vom so genannten Facharztstandard geprägt. Angemerkt sei bei dieser Gelegenheit, dass der Wortlaut dieser Norm einerseits zu weit und andererseits zu eng geraten ist. $\mathrm{Zu}$ wenig verlangt die Norm aus folgendem Grund: Es ist zwar seit jeher anerkannt, dass aufgrund des objektiv-typisierten Sorgfaltsstandards eine Unterschreitung dieses Standards nicht haftungsbefreiend wirkt. Anders liegt es aber bei demjenigen, der aufgrund einer besonderen Expertise oder besonderer Fähigkeiten mehr als das „allgemein Anerkannte“ zu leisten in der Lage ist. Der Arzt hat zu Gunsten seiner Patienten alle ihm zur Verfügung stehenden Fähigkeiten einzusetzen, er darf sich keineswegs unter kühlem Hinweis auf

10 Im Strafverfahren spricht man auch nicht von Parteien, sondern vom Beschuldigten, Angeschuldigten oder Angeklagten. 
die eben zitierte Vorschrift bei der konkreten Behandlung auf das Normalmaß zurückziehen, obwohl die Gegebenheiten und seine eigenen Fähigkeiten mehr zulassen ${ }^{11}$. $-\mathrm{Zu}$ viel verlangt umgekehrt die neu formulierte Norm, weil in bestimmten Situationen die Grenze von ,allgemein anerkannten fachlichen Standards" auch ohne entsprechende Vereinbarung bis zur Grenze des noch Vertretbaren unterschritten werden darf. Man denke an die Situation, dass bei Zeugen Jehovas die Vergabe von Frischblut so weit wie möglich hinausgezögert wird, was im Zweifel eine - vermutlich dem Willen solcher Patienten entsprechende - Unterschreitung des „allgemein anerkannten" Facharztstandards nach sich zieht ${ }^{12}$.

Wie dem auch sei: Abgesehen von derartigen Sonderfällen ist von einem Gericht in Bezug auf die gewöhnliche Behandlung der medizinische Standard zu Grunde zu legen. Dazu wird vielfach ein Sachverständiger (regelmäßig ein Mediziner) gehört. Er hat den allgemein anerkannten medizinischen Standard darzulegen und sich dazu zu äußern, ob und inwieweit der in Anspruch genommene Arzt davon abgewichen ist, auch, ob es für eine solche Abweichung medizinische Gründe gibt. Noch weitergehend wird zunehmend im Schrifttum diskutiert, den medizinischen Standard durch Leitlinien der Wissenschaftlichen Medizinischen Fachgesellschaften prägen zu lassen. Dabei werden drei verschiedene Stufen von Leitlinien unterschieden: S 1-Leitlinien beruhen auf einem informellen Konsens mit Empfehlungscharakter; S 2-Leitlinien werden aus formal bewerteten Aussagen der wissenschaftlichen Literatur entwickelt oder in einem bewährten formalen Konsensusverfahren verabschiedet; S 3-Leitlinien sollen alle Elemente systematischer Entwicklung aufweisen; hier ist gewissermaßen die höchste Entwicklungsstufe einer Leitlinie erreicht. Derartige S 3-Leitlinien sollen nun den Standard definieren. Sie gewännen damit den Charakter einer Rechtsnorm, indem sie im Prinzip verbindlich den Standard und damit den ärztlichen Pflichtenkatalog fixieren ${ }^{13}$. Gegen diese Einschätzung spricht m.E. nun ganz entscheidend der Umstand, dass es sich bei der Bildung entsprechender Leitlinien, die von Medizinern erstellt werden, keineswegs nur um einen Akt der Erkenntnis von Tatsachen handelt. Vielmehr

11 BGH NJW 1987, 1479: „Verfügte der Erstbeklagte [Chefarzt der chirurgischen Abteilung eines Krankenhauses] darüber hinaus [Standard eines erfahrenen Facharztes] über ärztliche, für die Therapie bedeutsame Spezialkenntnisse, dann hatte er sie auch zu Gunsten des Klägers einzusetzen." Anders Manfred Löwisch; Georg Caspers, in: Staudinger, BGB, Bearbeitung 2009, $\$ 276$ BGB Rdnrn. 29, 30.

12 Beispielhaft BVerfG NJW 2002, 206 und - in derselben Sache - OLG München MedR 2003, $174 \mathrm{~m}$. Anm. Bender (Blutübertragung erst nach Eintritt einer lebensbedrohlichen Situation).

13 Näher u.m.w.N. Spickhoff, in: Lilie/Bernhard/Rosenau (Hrsg.), Standardisierung in der Medizin als Rechtsproblem, 2009, S. 119, $126 \mathrm{ff}$. 
sind dem Prozess der Bildung von Leitlinien wertende Elemente eigen. Im Bereich von sogenannten Beratungsleitlinien wird das sofort deutlich, man denke etwa an Details einer nach ärztlicher Ansicht gebotenen oder eben auch nicht gebotenen Aufklärung. Gewiss gibt auch insofern je nach Sachlage erst genuin medizinischer Sachverstand Auskunft darüber, ob es z.B. überhaupt eine alternative Behandlungsmöglichkeit gibt, oder ob bestimmte, potentiell aufklärungsbedürftige Risiken dem fraglichen Eingriff anhaften. Ob aber ein bestimmtes Risiko gelaufen werden darf oder ob ein Patient über ein bestimmtes Risiko aufgeklärt werden muss, das sind Fragen, deren Beantwortung den Bereich der Wertung berührt und den der bloßen Tatsachenermittlung verlässt. Es handelt sich insoweit um eine genuin juristische Aufgabe, die im Prozess nicht einem (medizinischen) Sachverständigen überantwortet werden darf.

Freilich ist die Grenzziehung zwischen Norm und Bewertung auf der einen Seite und den tatsächlichen Grundlagen, die für die Subsumtion erforderlich sind, auf der anderen Seite keineswegs immer so eindeutig wie eben möglicherweise insinuiert. Das zeigt beispielsweise die Frage nach der Schuldfähigkeit oder Schuldunfähigkeit. Sie wird oft im Prozess ohne weitere Zwischenschritte an psychiatrische Gutachter gestellt. Das wird zwar vielfach kritisiert. Doch hat etwa Hans-Ludwig Schreiber mit guten Gründen bezweifelt, ob sich insoweit eine scharfe Trennlinie zwischen ärztlicher und juristischer Beurteilung ziehen lassen kann. Seiner Ansicht nach ist eine „normative Abstinenz" des Psychiaters und dessen Beschränkung auf angeblich rein tatsächliche Feststellungen von der Sache her gar nicht möglich. Ärztliche und juristische Beurteilung gehen eben doch manchmal ineinander über und lassen sich nicht immer säuberlich voneinander trennen ${ }^{14}$. Ungeachtet dessen ist unstreitig, dass der Richter die entsprechenden Ergebnisse des Sachverständigengutachtens keineswegs einfach übernehmen und sich ihnen anschließen darf, sondern er hat sich mit dem Gutachten seinerseits kritisch auseinander zu setzen und kann durchaus begründet - von den Ausführungen des Sachverständigen abweichen.

Schon durch derartige Relativierungen wird aber deutlich, dass wir als Juristen im Verhältnis von Recht und Medizin Schwierigkeiten haben, klare Grenzen zu ziehen. Umso mehr ist es angezeigt, sich durch einen Blick über die eigenen fachlichen Grenzen hinaus der eigenen Wertung zu vergewissern oder diese kritisch zu befragen.

14 Hans-Ludwig Schreiber; Henning Rosenau, in: Venzlaff/Foerster (Hrsg.), Psychiatrische Begutachtung, 4. Aufl. 2004, S. 85. 
Das sei an einigen herausgegriffenen Beispielen verdeutlicht. Man diskutiert etwa über den Einfluss von Beschränkungen im System der gesetzlichen Krankenversicherung auf den medizinischen Standard. Man sieht sofort, dass dadurch (und erst recht, wenn man das Beispiel auf die Frage nach der Fortsetzung oder den Abbruch lebenserhaltender Maßnahmen zuspitzt, wenn es um Fragen des Embryonenschutzes, der Klonierung usw. geht) ethische Fragen aufgeworfen werden, zu deren Beantwortung nicht nur die disziplinären Grenzen der Juristerei in Richtung auf die Medizin überschritten werden müssen, sondern zu deren Beantwortung wir andere Disziplinen zu Rate ziehen sollten, neben der Philosophie und der Theologie gewiss auch die (Gesundheits-) Ökonomie. In der von Hause aus interdisziplinär besetzten Akademie erhoffe ich mir hierzu manche Anregung und Kontakte, über den Gegenstand des Medizinrechts natürlich auch über die historisch-philologische Klasse hinaus. Nicht nur in Bezug auf die lex ferenda, sondern ebenso bei der Ausfüllung vorhandener, durchaus wertungsoffener Normen bietet jede Rechtsordnung, jedenfalls die deutsche, zureichend Spielräume, um sich Erkenntnissen anderer Disziplinen zu öffnen.

\section{III.}

Ich wende mich nun einem weiteren Bereich der Grenzüberschreitung zu, diesmal allerdings im schlicht geographischen Sinne. Juristen sind bekanntermaßen anders als andere Disziplinen (einschließlich der Medizin) von einer prinzipiell territorial begrenzten Rechtsordnung geprägt. Nur wenige beschäftigen sich mit Fragen des (bereits angesprochenen) Internationalen Privatrechts als Rechtsanwendungsrecht in Fällen mit Beziehungen zu mehr als einem Staat. Gerade im Bereich der Medizin finden wir in rechtstatsächlicher Hinsicht indes immer mehr solcher Fallkonstellationen, man denke an kostengünstige kosmetische Operationen im Ausland oder an den Versuch, durch Ein- oder Ausreise an einen höheren medizinischen Standard zu gelangen. Solche Phänomene werden mit den Schlagworten vom Medizin-, Ärzte- oder Patiententourismus im Zeitalter (auch) globalisierter Arbeitsmärkte nur unvollkommen charakterisiert.

An der Relevanz derartiger Themen ändert sich prinzipiell nichts dadurch, dass wir derzeit (auch) im Gesundheitssystem zunehmend ein Anwachsen eines - so gesehen übernationalen, vereinheitlichenden - Normenbestandes innerhalb der Europäischen Union zu konstatieren haben; als Beispiel sei die Richtlinie über die Ausübung der Patientenrechte in der grenzüberschreitenden Gesundheitsversorgung vom 09.03.2011 ge- 
nannt ${ }^{15}$. Denn auch die EU-,,Gesetzgebung“ hat räumliche Grenzen. Sie prägt keineswegs per se den juristischen „Weltstandard“, ist also zumindest rechtspolitisch zu hinterfragen. Dazu bietet auch die Rechtsvergleichung (im Sinne der Vergleichung wesentlicher nationaler Regelungsmodelle) viele und oft weiterführende Hilfestellungen und Anregungen. Schon innerhalb Europas bedürfen Normen der EU der europäisch-rechtsvergleichend unterfütterten Ausfüllung. Dazu ist dann freilich vorrangig auf die nationalen Rechtsordnungen der EU-Mitgliedstaaten abzustellen. Dass Regeln der EU rechtspolitisch keineswegs unfehlbar oder auch nur unabänderlich sind, zeigt der Plan für eine neue EU-Verordnung zu klinischen Studien, durch die die geltenden EU-Richtlinien hierzu abgeändert werden sollen. Insbesondere wird angestrebt, bürokratische und finanzielle Hemmnisse abzubauen. Hintergrund für diesen Plan ist nicht zuletzt, dass die Zahl der klinischen Prüfungen für Arzneimittel in den vergangenen fünf Jahren innerhalb der EU um ein Viertel gesunken ist. Gleichzeitig hat (was kein Geheimnis ist) in erheblichem Ausmaß eine Verlagerung von Studien in Drittstaaten stattgefunden, in denen wohl nicht nur die juristischen Standards nicht die hiesigen erreichen ${ }^{16}$.

Rechtstechnische Antworten auf die damit aufgeworfenen Fragen erfordern neben juristisch-handwerklichem (dogmatischem) Nachdenken auch ethische Grundentscheidungen unter Einbeziehung der Analyse von ökonomischen Wirkmechanismen. Wie weit soll man einer Flucht aus europäischen Standards (nicht nur) im Kontext der medizinischen Forschung am Menschen durch eine Absenkung der eigenen Standards begegnen? Sind dabei faktische Grenzen einer Durchsetzung europäischer, eigener Standards auch im ferneren Ausland in Rechnung zu stellen? ${ }^{17}$ Ethisch Wünschenswertem sind Grenzen der schlichten Durchsetzbarkeit, der eigenen Macht gesetzt; das Völkerrecht hilft in diesem Bereich wohl nur begrenzt weiter.

15 ABI EU, S. L 88/45.

16 Siehe den Bericht von Petra Spielberg, EU-Verordnung klinische Studien: Mehr Kontrolle, weniger Bürokratie, Deutsches Ärzteblatt 2012, A-1752.

17 Ein Beispiel für den Versuch einer kaum gelungenen Erstreckung europäischer Standards auf ausländische Staaten findet sich in Art. 25 des Zusatzprotokolls zum Übereinkommen über Menschenrechte und Biomedizin über biomedizinische Forschung des Europarates; siehe dazu Hans-Christoph Kandler, Rechtliche Rahmenbedingungen biomedizinischer Forschung am Menschen, 2008, S. 267 ff. 


$$
I V
$$

Nach dem Streifzug durch verschiedene Ebenen der Grenzziehungen und Grenzüberschreitungen im Kontext von Medizin und Recht und auf diese Weise durch wichtige meiner Arbeitsfelder habe ich mich an die allseits bekannte „Rangordnung“ Immanuel Kants in seinem Streit der Fakultäten ${ }^{18}$ erinnert. Die Juristen bleiben sowohl in der theoretischen wie in der praktischen Rangordnung Kants (in der bekanntlich nur die Theologie, die Medizin und die Rechtswissenschaft vorkommen) konstant auf dem mittleren, zweiten Platz und stehen insoweit also immer in der direkten Nachbarschaft von beiden. Da Juristen anders lautenden Gerüchten zum Trotz lernfähig, ja sogar wissbegierig sein können, suchen wir, um unserem Ziel der Herstellung irdischer Gerechtigkeit möglichst nahe zu kommen, jedenfalls an der Universität gerne den Sachverstand und Rat nicht nur benachbarter Rechtsordnungen, sondern auch benachbarter Disziplinen, ganz gleich, ob diese nach Kant nun theoretisch oder praktisch „über“, „unter" oder (wie es sich gehört) kollegial neben uns stehen.

18 1798, Nachdruck philosophische Bibliothek Band 252, 1959, S. 14. 